\title{
Analyzing Web Site Performance between Edge Computing and Cloud Computing
}

\author{
Waldon Hendricks ${ }^{1}$ \\ ${ }^{1}$ Cape Peninsula University of Technology
}

June 25, 2021

\begin{abstract}
Developments such as the Internet of Things and how it integrates with cloud computing statistics show that latency is one of the issues that is inconsistent. Having more devices connect to the internet every year brought about a newer technology called Edge computing. This study aims to prove that Edge computing that brings computing resources closer to the edge of the network, improves latency. The method used for this research was that of an experiment. Analyzing the latency figures between Edge and Cloud computing is the purpose of this study, by making use of 2 hosted services, hosting a web site that will be load tested to provide the latency figures. Edge computing proved through the statistics to be more beneficial in improving latency. Due to time constraints, virtual users and hosted services were used.
\end{abstract}

\section{Introduction}

In the last decade organizations and individuals became dependent on computer technologies that use cloud computing. These technologies improve business processes, synchronize smart devices, vehicle tracking, digitalise collections of data, as well as for big data storage. By the year 2020, approximately 50 million devices will be connected to the internet, according to Cisco (Afshar, 2017). Currently, the Internet of Things is on a rise, with applications demanding real-time interactivity and devices that are producing huge amounts of data to and from the cloud. Cloud services today houses state of the art applications making it easy to use, provides flexible scalability, and with an understanding that resources are that of an infinite nature (Ni et al., 2018).

When it comes to technology there are always drawbacks to the much-anticipated benefits (Bermbach et al., 2018). Cloud research has grown in the last decade with edge computing (EC) brought forth as a solution to conventional centralized computing to promote decentralized computing (Cao et al., 2018). Thus providing Edge Computing, providing benefits in terms of performing computing needs close to the source, where data is generated to bring about service benefits. But does edge computing provide a means to lower latency, provide more accurate statistics and improve security with regards to existing websites and how it is provided to end-users? (Bilal et al., 2018).

Salman et al. (2016) state that edge computing is a solution, exports some of the clouds' current capabilities closer to the user, decreasing latency. Another benefit stated is that due to the increasing time-sensitive Internet of Things (IoT) applications on the rise, more areas in IoT can be reached. More benefits such as 
increased security are included with edge computing as the number of hops on the network is less minimizing man-in-the-middle attacks for example (Yi et al., 2015).

Luan et al. (2015) state that around $90 \%$ of the users around the world depend on cloud services. Having more users looking towards making use of cloud services to host businesses and wanting the business to be successful looking at the performance of it would be a great place to start. Edge computing can be done on many devices such as routers, switches, other network management devices, and even smartphones, all these devices would need is to be equipped with enough processing power and storage to act as an edge device (Cao et al., 2018).

With concerns about security, edge computing brings better protection to end-users by keeping personal data at the edge network and only sending well-organized data to the cloud (Bermbach et al., 2018). Edge computing has been proven to provide a more secure infrastructure due to its local data storage or personal data and non-real time of exchange of data with the cloud (Ni et al., 2018). Local edge nodes (also known as mini-clouds) provide extra security to end-points, by acting as proxies to assist in managing and updating credentials, thus taking away depending on the cloud for such impractical needs.

Researching the latency benefits edge computing has over cloud computing is what this research paper sets out to achieve. This research paper will focus on analyzing the latency figures between edge computing and cloud computing to see if the latency advantages are what literature says they are.

\section{Background to the study}

In the years leading up to 2019 , we have had hundreds of powerful computing devices connect to the internet through different types of network connections. The rise of the Internet of Things and how it generates and transmits huge amounts of data to the cloud almost every second, it consumes bandwidth and increases latency as well as bringing properties of Big Data with it. With more devices being connected to the internet in the future, current infrastructures will become inundated with the increase in users placing strain on resources.

Cloud computing has had a crippling factor that has not really been solved which would be the unreliable latency and lack of mobility to name a few (Yi et al., 2015). Reddy et al. (2011) state that the latency on cloud computing must be improved as the large bottlenecks lie within the storage and memory.

Lin et al. (2007) state that edge computing's emergence has been an important one to deliver Web content over the internet as the internet continues to grow explosively.

When it comes to websites, especially e-commerce, many companies struggle when they host certain promotions and the amount of user traffic on such days end up crippling websites. On a normal day, the amount of users logging in and using the website poses no threat but as soon as there is an influx of users per second, latency becomes effected and speeds drop to the extent where a user will not gain access to the website. Users can have the best internet connection or internet speeds when accessing a website, it is the company's infrastructure that determines how well the website performs. Poor performance results can not only result in unhappy customers but a loss in sales. 


\section{Problem Statement}

With more users connecting to the internet consuming resources when browsing, better performances will be needed. The constant connectivity and real-time interactions between networks and cloud latency being high, as well as security concerns with the number of hops that take place on a network to reach a website, could edge computing provide the needed benefits? Users being impacted by experiencing delays in real-time data exchange and slow performances on websites could be catastrophic depending on the application being used and the purpose thereof. So henceforth, the purpose of this paper is to establish if Edge Computing is a possible solution to that of Cloud Computing and if the latency is more efficient in the latter?

\section{Research Questions}

\section{Research question 1}

How does edge computing compare the benefits of improved latency compared to conventional cloud computing?

\section{Research Question 2}

Which hosted service, cloud computing or edge computing handles a load testing of users better?

\section{Aims and Objectives}

The study aims and objectives of this research are determined as follows:

\section{Aim}

To establish if edge computing improves network latency compared to using a website hosted on the cloud

\section{Objectives}

1. To examine latency speeds in an edge computing scenario and in a cloud computing scenario

2. To establish if it is more beneficial to invest in edge computing than cloud computing

\section{Research Design and Methodology}

Research methodology can be described as a set of procedures and methods that help researchers structure and carry out the research at hand objectively (Kothari, 2004). There are research methods and there are research strategies, where research methods are defined as the tools that are used to do the research, research strategies are more like the instruments that have to be well planned and thought out to record, process and observe data (Rajasekar et al., 2013). In academic research nothing is simple and every decision and step has to be well planned, thought out and chosen accordingly (Greener, 2008).

The research methods are described as tools to conduct research systematically, there are also the research techniques that include the tools to perform the research, and either observe or gather the data, record the data and process the data into useful information (Rajasekar et al., 2013). Research can be defined as a search for knowledge, but also as a scientific and systematic investigation for information on a specific topic (Kothari, 2004). Not only does research enable an investigation for knowledge but an effort to gain new knowledge and new facts. 
Research strategies are to be used and can be defined as the well thought out plan on how to conduct the research. This would include the research types and how the data produced from the research will be collected and analyzed. The information for the research can be collected from various sources such as books, people, journals, experience, observations, and so on (Rajasekar et al., 2013). There are in fact a few questions that should be asked when considering what type of research methodologies are to be used, such as:

- Which of the methods are most suitable for the problem identified?

- Is it in-depth research or a high volume of measurable data?

- What kind of data is the researcher wanting to achieve?

- When choosing a method, how accurate will it be in analyzing the results?

When starting with the data part of a research study, the research question should be to help in choosing the correct data strategy, data generation method, and data analysis to be done for the research. The data strategies consist of options such as case studies, experiments, ethnography studies, and design and creation to name a few (Creswell, 2003). The type of data that will feed the strategy can be chosen from a pool of generation methods such as observations, questionnaires, and interviews to name a few (Neville, 2005).

The three approaches to data analysis that are considered for research being performed are either qualitative, quantitative or mixed methods (combining both the qualitative and quantitative methods). A qualitative approach is an unstructured approach and the quantitative approach relates to a structured approach (Kumar, 2011). A qualitative study is used when the study is primarily described as either a situation, problem or phenomenon. Qualitative measures would be used to gather information on the variables. A quantitative study relates to quantifying the variations in a situation or problem or issue, information is gathered by using quantitative variables (Neville, 2005).

Quantitative research uses statistics drawn from the variables of the study but the use of statistics is to either confirm or contradict the conclusion drawn at the basis of understanding the data, whereas qualitative research is more subjective and involves looking at less tangible aspects of research (Neville, 2005). The quantitative approach also deals with positivist knowledge claims while qualitative deals with constructivist knowledge claims (Greener, 2008). The mixed-methods approach would bring pragmatic knowledge claims, making use of both quantitative and qualitative data analysis sequentially.

Quantitative research is in nature more of an ordered approach than what qualitative research is and the research of quantitative is expressed with numbers (Creswell, 2003). The qualitative approach is more focused on the assessments of attitudes, opinions, and behaviour.

Addressing the objectives and questions detailed earlier in this research paper, this section will cover the appropriate research methodologies to be used for the completion of this type of study.

\section{The method chosen for the research at hand}

Understanding the research and what is required for the research to reach completion, the correct data strategy, data generation method and data analysis have to be chosen. For this research, when looking at the research questions, the considerations of what can generate the best data for this research would be to observe either certain situations or scenarios. How is this to be performed? 
The strategy chosen for this research was an experiment. The reason for going with an experiment is because it will best achieve the data required, testing two different architectures with literature stating that edge computing provides lower latency than that of cloud computing. To analyze the data, a quantitative approach was used to look at the data figures collected, which was performed on a small scale but also allowing the data to test the difference between the chosen variables.

Kothari (2004) mentions the use of an experiment data generation method, testing an independent variable against a dependent variable, observing the different results and making claims from the conclusions. The experiment consisted of setting up a cloud server and an edge server to test how the two instances of the computing architectures handle latency on load (by simulating many users to use the website). Both of the architectures hosted a website and load testing was performed on both of the instances to observe how latency is handled. While the experiments took place, observations were done on the variables and the outcomes noted providing the data needed for this research. Comparing the use of cloud computing and edge computing, provided the answers to the proposed research questions.

Data analysis took place using quantitative methods. After reading the comparison between quantitative and qualitative methodologies, the decision of using quantitative methods for the data analysis as the study requires an understanding of how latency is affected by different amounts of users accessing the website at once in the scenario. Choosing quantitative as the data analysis as the data generated is statistical and has a prediction, unlike qualitative that seeks to understand a phenomenon.

A deductive approach to this research is used as it makes use of using literature or theory and a prediction that edge computing has lower latency formed the theory that is put to the test (Greener, 2008).

The data gathered for this research came from an experiment by making use of free trials for the cloud and edge instances, open-source software, as well as an internet connection, to test both of the scenarios, which will be latency the latency figures. The source of the results for this research would be primary in nature as it is data that would be generated from an experiment and not sampled or collected from previous findings.

Quantitative research will not only explore an issue through one perspective but gather different views for the problem to be researched and understood (Baxter \& Jack, 2008). The research will bring about awareness of the potential that edge computing has to offer organizations.

With the time frame available towards this research, generating, gathering and analyzing the data, an experiment will be more beneficial in saving the costs to time. Gathering the experts in the field and structuring the interviews or surveys will consume more time to send around and conduct. The data generated from the experiment will not contain any bias as there is no preferred approach from the researcher (Greener, 2008). There is no audience in this research and there is no subject group or group of participants only technologies that will be used to generate the data needed for this research by the researcher.

\section{Conceptual Frameworks for the Experiment}

The following conceptual framework was created as a guide to follow when construction the the experiment to test the types of computing: 


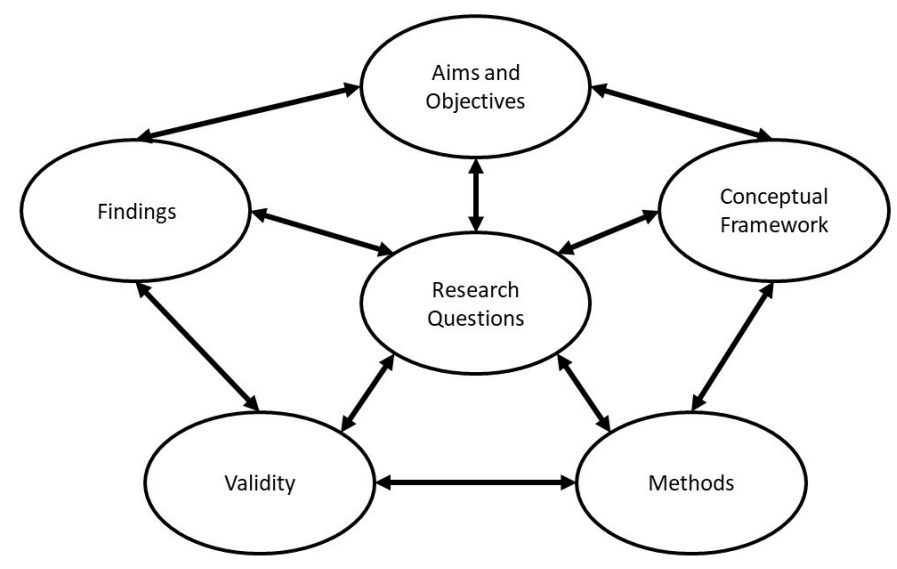

Figure 1: Computing conceptual framework

In order to address the research questions, meeting the aims and objectives this paper sets out to archive, the conceptual framework constructed for the experiment depicts how the correct validity of the findings link to the methods and the questions.

The following conceptual framework models were created to assist in designing and testing the latency between cloud computing and edge computing.

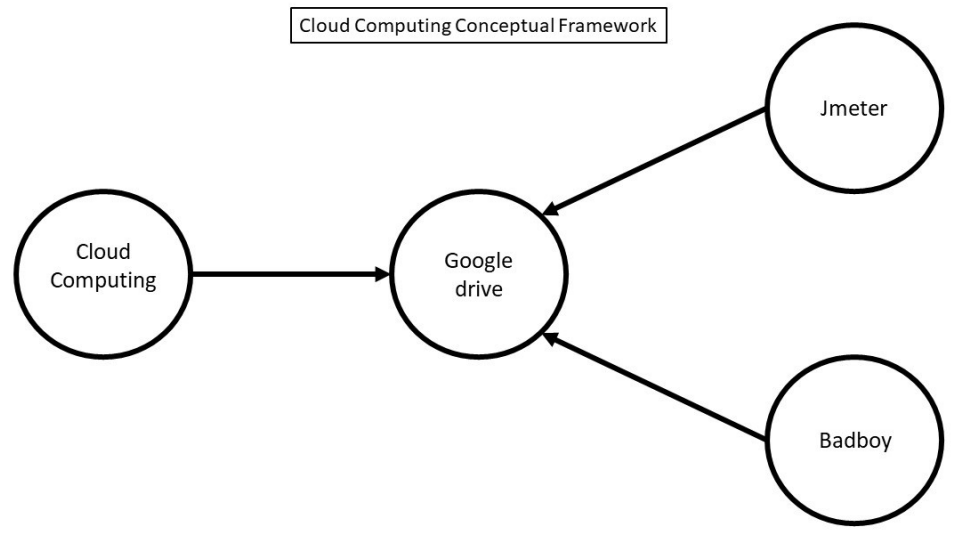

Figure 2: Cloud computing conceptual framework

Looking at the literature gathered, Figure 2 displays an overview of how the data will be collected when making use of cloud computing. A hosted service will be used, which is a google drive. The testing tools such as Jmeter and Badboy will be used to both create a number of virtual users as well as execute the users 
against the google drive.

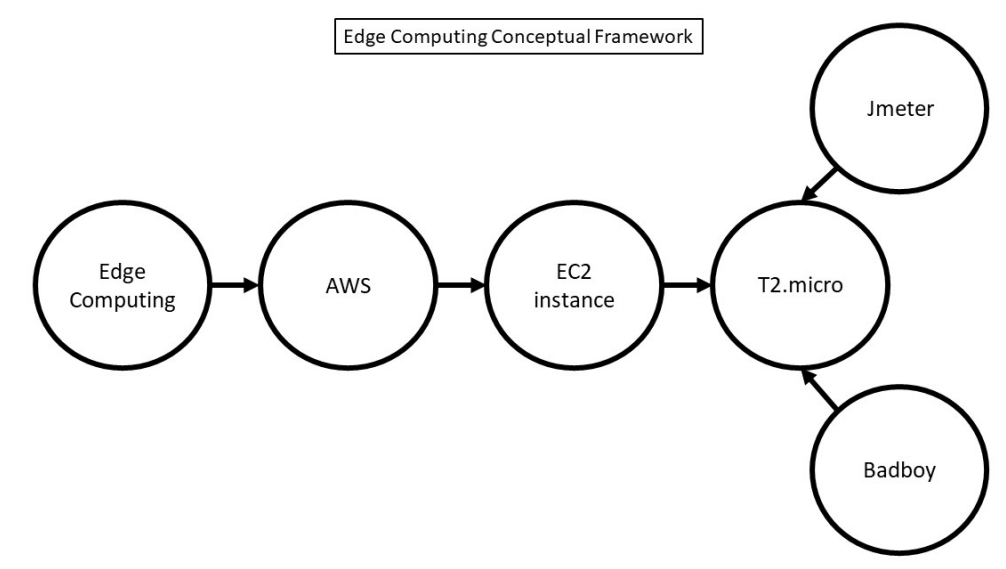

Figure 3: A line graph showing scenario 1 with clouds latency figures

The edge computing part of this test will also make use of a hosted service provided by Amazon Web Services, which is an EC2 instance. There are various option in infrastructure do choose from but for this experiment the t2.micro was chosen as a free hosted service. The same tests will be performed by using Jmeter and Badboy to create and simulate users. The hosted service can be swapped out and the same scenarios can be replicated to compare the better-hosted service.

\section{Data collection, analysis and findings}

The decision was made to make use of an experiment was because the data being collected for this research had to result from testing findings in the literature that showed the benefits of edge computing over that of cloud computing. The data collected are the times taken from how long it takes for interaction between a computer network to a website and back or the simulation of it, this known as the latency. The latency was measured between a cloud instance and an edge instance.

The solution to latency in this case, due to the findings from literature would suggest that edge computing is faster at computing than cloud computing. The number of users will be controlled by the experimenter, making this the independent variable in the experiment, with the dependent variable being the latency and how increasing the load of users per session affects the latency on the instances.

When collecting the data, it was important for the data to be as reliable as possible and unbiased as literature states that the newer technology edge computing will be the way forward by computing data at the edge of the network. The data collected is primary data and collected from running the experiment scenarios from the same computer and network to the two instances to achieve the most accurate set of results.

The research at hand required an experiment or an observation to achieve the needed results, at the end of the day an experiment was chosen to study the latency figures and how the different computing paradigms handled the swarm of users. 
Due to the costs and time available for this research, the experiment was done using hosted services from Google and Amazon Web Services. The offering of free trials and open source software made the experiment achievable within the given time frame, as well as show the capabilities of that these services have to over whether it be cost-driven or performance-driven.

\section{Data Collection}

A google drive hosting a website in the cloud will be scenario 1 and an Amazon Web Services (AWS) Elastic Compute Cloud (EC2) instance hosting a WordPress website will be used to illustrate edge computing which will be scenario 2. Both google drive and AWS are used on a free trial basis with Jmeter and Badboy being open source. Ethics were taking into account as not to exploit or breach any policies of any kind.

Badboy that is used for web testing and development testing is used to create a capture replay script that functions as a user manoeuvring to and using the website. Supplying Badboy with the URL to the website point the test to the website while recording every movement. Using Badboy automation script creator to create a test script that automates the action of a user, the script was used in Jmeter to run against both the cloud-based website and the edge website to produce the latency times. Jmeter helps to set how many users each test will consist of and in what time frame it should run amongst other variables that are not needed for this experiment. Within Jmeter an HTTP test plan can be created as well to perform the load test but they both yielded the same latency results. The websites URL link was supplied and the test would run as if it were a user accessing the website. Using 1 user as the benchmark to document what the latency was on the network, more users were then added to form the load tests.

Using Jmeter in both scenarios, increasing the number of users to affect the latency figures. From running the session with 1 user, the experiment is then increased from 1 user to 10 then 25, 50, 75, 100 and ending at 1000 users. All users are set to run within 1 second thus simulating a load scenario to consume both bandwidth and affect latency. Using more users in the shortest time available would illustrate the power edge computing has over cloud computing when a website experiences a spike in traffic.

The AWS EC2 instance hosted a website similar to that hosted on the google drive to achieve the most accurate results for this experiment. The way in which the first test was run, with incrementing the users up unto 1000 users are followed to the same extent on the second experiment as the first. Also, making use of the same network, same computer and test times run was a must to ensure similar scenarios for close comparisons. The differences in the experiments came to how one computing was set up in comparison to the other, and that yielded different results.

After conducting the experiment, two sets of figures for each experiment was achieved. First beginning with the cloud latency experiment:

\section{Cloud Computing Experiment}




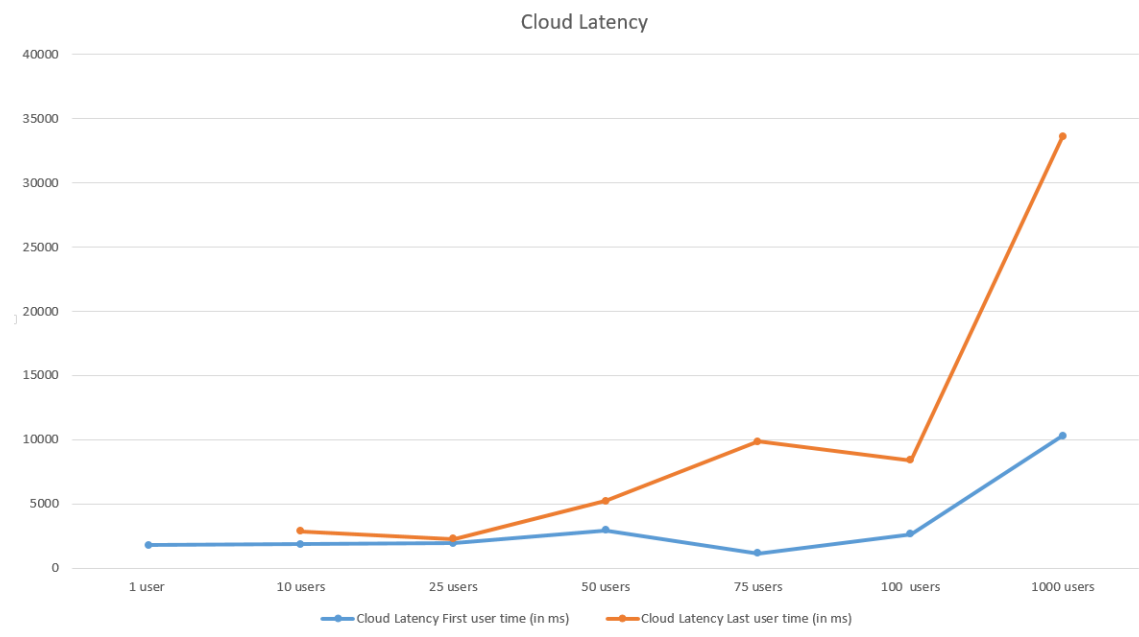

Figure 4: A line graph showing scenario 1 with clouds latency figures

\begin{tabular}{|c|c|c|}
\hline \multicolumn{3}{|c|}{ Cloud Latency } \\
\hline Number of weer(s) & Fut weer time ( $\mathrm{n} \mathrm{mo}$ ) & Lant ueer time (in me) \\
\hline 1 ueer & 1773 & \\
\hline 10 ues: & 1872 & 2886 \\
\hline 25 ueers & 1924 & 2286 \\
\hline 50 ueers & 2961 & 5211 \\
\hline 75 uect & 1152 & 9847 \\
\hline 100 ueer: & 2643 & 8395 \\
\hline 1000 ueer: & 10307 & 33627 \\
\hline
\end{tabular}

Figure 5: The difference in latency times when the first user communicates with the website and the last user communicates on the busy website on scenario 1

The line graph 4 takes 2 types of users into account, the first user which starts of the load and the last user enters at the busiest part of the test while the website is congested with users. The blue line illustrates how busy the network becomes while the first few users begin the session illustrating a spike in latency on a busy peak of users. The orange line does not achieve the consistency that the first users appear to achieve as it reacts to the different types of stress from the load test. The first user in a normal scenario achieves a latency time of 1773 milliseconds almost staying constant until it reaches 50 users where it spikes to 2961 milliseconds to drop to 1152 milliseconds at 75 users. At 100 users using the website, the first user peaks at 2643 milliseconds, reaching a high of 10307 milliseconds for the first user and the last user in that session reaches 33627 milliseconds, which is approximately 3 times that of the first user.

Edge Computing Experiment 


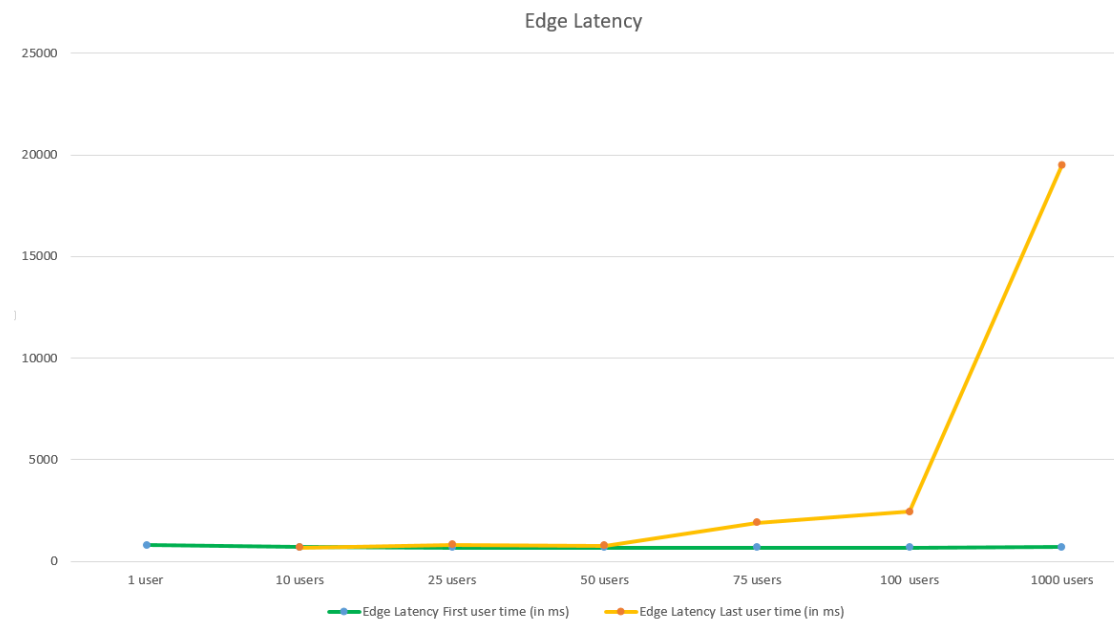

Figure 6: Line graph depicting the Edge latency figures

\begin{tabular}{|c|c|c|}
\hline \multicolumn{3}{|c|}{ Edge Latency } \\
\hline Number of ues(s) & Frit ueer time ( $(\mathrm{n} \mathrm{ms}$ ) & Last ueer time (n me) \\
\hline 1 weer & 797 & \\
\hline 10 ueer: & $\mathbf{7 1 0}$ & 691 \\
\hline 25 ueers: & 671 & 812 \\
\hline 50 ueer: & 692 & 776 \\
\hline 75 ueer: & 678 & 1905 \\
\hline 100 ueers & 669 & 2453 \\
\hline 1000 ueer: & 703 & 19513 \\
\hline
\end{tabular}

Figure 7: The difference in edge computing latency times for the first user and the last user entering the website for scenario 2

\section{Discussion and interpretation of findings}

The data collected for this research shows that the edge scenario provides an improvement in latency to that of the cloud. Not only was the latency improved but the overall time it took to run each test was much shorter than on the edge instance compared to the cloud instance.

The line below comparing the latency between cloud and edge scenarios shows that each test on the edge scenario remained more constant in its latency and run times that the cloud scenarios inconsistencies in latency. 


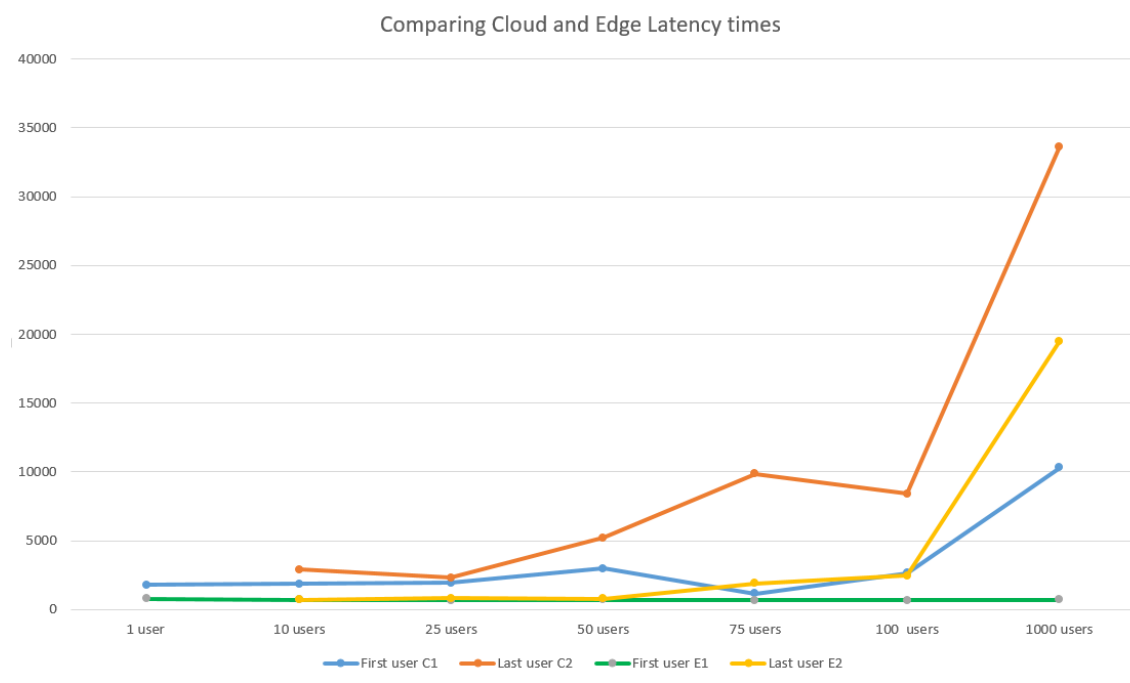

Figure 8: Line graph displaying both the edge and the cloud computing latency times

The experiment of testing the latency differences between the cloud and edge computing paradigms was successful in proving that the edge website provides better latency figures over the cloud website as shown in fig 6 . In the line graph fig 6 , comparing both scenarios with the blue and orange line belong to the cloud, and green and yellow belonging to the edge, the differences between the first and the last user in milliseconds are big.

The figure below shows the total run times of each scenario and how it performed:

\begin{tabular}{|c|c|c|}
\hline \multicolumn{3}{|c|}{ Run Trme: } \\
\hline Number of ueer(s) & Cloud ru time & Edge ru time \\
\hline 1 ueer & 3 seconds & 1 second \\
\hline 10 ueer: & 5 seconds & 1 second \\
\hline 25 ueer: & 10 seconds & 1 second \\
\hline 50 ueer: & 17 seconds & 1 second \\
\hline 75 ueers & 34 seconds & 2 seconds \\
\hline 100 ueer: & 25 seconds & 3 seconds \\
\hline 1000 ueers: & $6 \mathrm{~min} 14 \mathrm{sec}$ & 20 seconds \\
\hline
\end{tabular}

Figure 9: Run times showing how long it took to run each latency test by the number of users used

Not only does the latency results show a lower latency measure but the durations in which each test is run is much shorter on the edge instance than it is with the cloud. It took 100 users 3 seconds to successfully communicate with the edge website and 25 seconds with the cloud. After testing more than 100 users the times would increase in duration on the cloud website than that of the edge, showing that the computing power and strength lie with edge networks. 
To answer the objectives, in summary, the latency is lower when it comes to using the edge architecture in comparison to the higher latency times on the cloud instance when more users were added to the experiment. If a company or organization, depending on the service it renders, expect to experience high volumes of traffic during certain times where it is deemed crucial, making use of edge architectures will bring performance benefits.

\section{Conclusions \& recommendation}

The research objectives and aims were reached, as well as answering the questions relating to the edge architecture and how it compares to cloud computing. Out of the experiments that were conducted, results show that the latency is much better-using edge computing instead of cloud computing as the increase in users had minimal effect on the latency figures. Literature states that edge computing does not only hold latency advantages but security advantages. The insights of how the data was collected were discussed and insights provided. With some limitations to the research, the goal of producing latency times using edge computing to compare against cloud computing was successful to reach the conclusion.

Future research suggestions could be using a much bigger edge architecture, created in anticipation of experiencing a high volume of users to archive more accurate results. Depending on the volumes of traffic expected for the type of service or purpose of the organizations looking to make use of edge computing, would be making a step in the right direction.

\section{Limitation of the Study}

Due to time constraints, a small edge network was created and used with a big enough virtual user pool size that the network could handle. The lack of experience to develop a virtual edge network also resulted in a limitation to this study, which leads to making use of free hosted services. There was also only one internet connection towards both the cloud and edge instances being used to allow the number of virtual users to load the instances, having different internet connections or sessions could also bring a change in results.

\section{References}

Afshar, V. 2017. Cisco: Enterprises Are Leading The Internet of Things Innovation. HuffPost : 1. https://www.huffingtonpost.com/entry/cisco-enterprises-are-leading-the-internet-of-things_us_59a41fcee4b0a62d0987b0c6.

Bandyopadhyay, D. \& Sen, J. 2011. Internet of things: Applications and challenges in technology and standardization. Wireless Personal Communications , 58(1): 49-69.

Baxter, P. \& Jack, S. 2008. The Qualitative Report Qualitative Case Study Methodology: Study Design and Implementation for Novice Researchers. The qualitative reportualitative report , 13(2): 544-559.

Bermbach, D., Pallas, F., Pérez, D.G., Plebani, P., Anderson, M., Kat, R. \& Tai, S. 2018. A Research Perspective on Fog Computing.Lecture Notes in Computer Science (including subseries Lecture Notes in Artificial Intelligence and Lecture Notes in Bioinformatics) , 10797 LNCS: 198-210. 
Bilal, K., Khalid, O., Erbad, A. \& Khan, S.U. 2018. Potentials, trends, and prospects in edge technologies: Fog, cloudlet, mobile edge, and micro data centers. Computer Networks , 130(2018): 94-120. https://doi.org/10.1016/j.comnet.2017.10.002.

Cao, J., Zhang, Q. \& Shi, W. 2018. Challenges and opportunities in edge computing. SpringerBriefs in Computer Science : 59-70.

Creswell, J.W. 2003. Research Design, Second Edition. Research Design, 2nd Edition : 3-26.

Dolui, K. \& Datta, S.K. 2017. Comparison of edge computing implementations: Fog computing, cloudlet and mobile edge computing. GIoTS 2017 - Global Internet of Things Summit, Proceedings .

Foster, I., Zhao, Y., Raicu, I. \& Lu, S. Cloud Computing and Grid Computing 360-Degree Compared.

Greener, S. 2008. Business Research Methods . http://search.proquest.com/docview/209900392?accountid=458\%5Cnhttp://li 06-01\&spage $=266 \&$ title $=$ Management + Learning\&atitle $=$ Business + Researc.

Gubbi, J., Buyya, R., Marusic, S. \& Palaniswami, M. 2013. Internet of Things (IoT): A vision, architectural elements, and future directions.Future Generation Computer Systems , 29(7): 1645-1660. https://arxiv.org/pdf/1207.0203.pdf?ref=theiotlist.

Kothari, C.R. 2004. Research Methodology - Methods and Techniques .

Kumar, R. 2011. Research Metolodogy Step by Step Guide for Beginners . http://www.sociology.kpi.ua/wpcontent/uploads/2014/06/Ranjit_Kumar-Research_Methodology_A_Step-by-Step_G.pdf.

Lin, Y., Kemme, B., Patiño-Martínez, M. \& Jiménez-Peris, R. 2007. Enhancing edge computing with database replication. Proceedings of the IEEE Symposium on Reliable Distributed Systems : 45-54.

Luan, T.H., Gao, L., Li, Z., Xiang, Y., Wei, G. \& Sun, L. 2015. Fog Computing: Focusing on Mobile Users at the Edge. : 1-11. http://arxiv.org/abs/1502.01815.

Miorandi, D., Sicari, S., De Pellegrini, F. \& Chlamtac, I. 2012. Ad Hoc Networks. Proceedings of the International Conference on IoT in Social, Mobile, Analytics and Cloud, I-SMAC 2017, 10(7): 1497-1516. http://dx.doi.org/10.1016/j.adhoc.2012.02.016.

Neville, C. 2005. Introduction to Research and Learning Service Research and. Bradford University : 1-5.

Ni, J., Zhang, K., Lin, X. \& Shen, X.S. 2018. Securing Fog Computing for Internet of Things Applications: Challenges and Solutions.IEEE Communications Surveys \& Tutorials , 20(1): 601-628. http://ieeexplore.ieee.org/document/8066283/.

Rajasekar, S., Philominathan, P. \& Chinnathambi, V. 2013. Research methodology. Contributions to Management Science : 1-53.

Reddy, K. V., Rao, T.B., Reddy, L.S.S., Kiran, S.P., Reddy, V.K., Rao, B.T., Reddy, L.S.S. \& Kiran, P.S. 2011. Research Issues in Cloud Computing. Online , 11(11): 10-12. http://computerresearch.org/stpr/index.php/gjcst/article/viewArticle/854.

Salman, O., Elhajj, I., Kayssi, A. \& Chehab, A. 2016. An architecture for the Internet of Things with decentralized data and centralized control. Proceedings of IEEE/ACS International Conference on Computer Systems and Applications, AICCSA , 2016-July(August 2016).

Shi, W., Cao, J., Zhang, Q., Li, Y. \& Xu, L. 2016. Edge Computing: Vision and Challenges. IEEE Internet of Things Journal , 3(5): 637-646.

TongKe, F. 2013. Smart Agriculture Based on Cloud Computing and IOT.Journal of Convergence Information Technology , 8(2): 210-216. http://www.aicit.org/jcit/global/paper_detail.html?jname=JCIT\&q=2598. 
Yi, S., Li, C. \& Li, Q. 2015. A survey of fog computing: Concepts, applications and issues. Proceedings of the International Symposium on Mobile Ad Hoc Networking and Computing (MobiHoc) , 2015-June(June 2015): $37-42$.

Zhang, Q., Cheng, L. \& Boutaba, R. 2010. Cloud computing : state-of-the-art and research challenges. : $7-18$. 